\title{
Pre-discharge Predicting the 5-years Hospital Readmission in Patients with Schizophrenia and Schizoaffective Disorders
}

\section{Yael Ratner ${ }^{1}$ and Michael Ritsner ${ }^{1,2 *}$ \\ ${ }^{1}$ Shaar Menashe Mental Health Center Affiliated to Rappaport Faculty of Medicine, Technion Israel Institute of Technology, Haifa, Israel \\ ${ }^{2}$ National Insurance Institute in Israel, Haifa, Israel}

*Corresponding Author: Michael Ritsner, Professor, National Insurance Institute in Israel, Haifa, Israel.
Received: September 25, 2021

Published: October 22, 2021

(C) All rights are reserved by Yael Ratner and Michael Ritsner.

\begin{abstract}
Background: Unplanned patient readmission (UPRA) is frequent and costly in healthcare settings. This study aimed to create a prediction model for predischarge detection of 5-years UPRA of patients with schizophrenia and schizoaffective (SZ/SA) disorders.

Methods: Consecutively admitted inpatients were comprehensively assessed before discharge from the hospital. After discharge, the readmission was tracked via computerized medical records for 5 years.

Results: Of 125 patients, $80.8 \%$ of participants were readmitted for the 5-year period (63.2\% had readmissions within the first year). Regression analyses suggest the following predischarge predictors of readmissions: previous hospitalizations, elevated suicide risk, sensitivity and hostility scores, while better satisfaction with the quality of life and social support, lower depression and paranoid ideations, good adherence to treatment decreased readmission rates. Sociodemographic, background, and clinical variables did not reach a significant level to be predictors. The logistic regression model correctly classified $83.1 \%$ of subjects by their readmittance status.
\end{abstract}

Conclusion: The study revealed predischarge predictors for 5-years readmissions, and underlines the importance of assessing patient-reported outcome measures to identify patients at risk of readmission to the hospital.

Keywords: Mental Disorders; Readmissions; Predischarge Predictors; Distress; Quality of Life; Social Support

\section{Abbreviations}

BARS: Brief Adherence Rating Scale; CGI - S: Clinical Global Impression-Severity Scale; GAF: Global Assessment of Functioning Scale; Mini - PANSS: Mini Positive and Negative Syndrome Scale; MSPSS: Multidimensional Scale of Perceived Social Support; OAS: Overt Aggression Scale; PROMs: Patient-reported Outcome Measures; PSRS: Plutchik Suicide Risk Scale; SZ/SA: Schizophrenia and Schizoaffective Disorders; TBDI: Talbieh Brief Distress Inventory;
Q-LES-Q - 18: Quality of Life Enjoyment and Satisfaction Questionnaire - Abbreviated Version QOL - Health-related Quality of Life.

\section{Introduction}

Patients with severe mental disorders have been recognized as a risk group for readmission to the hospital. Unplanned patient readmission continues to attract considerable attention because of its substantial negative influence on patients' quality of life and

Citation: Yael Ratner and Michael Ritsner. "Pre-discharge Predicting the 5-years Hospital Readmission in Patients with Schizophrenia and Schizoaffective Disorders". Acta Scientific Neurology 4.11 (2021): 39-52. 
healthcare costs. The identification of patients at high risk for readmission after is a logical first step in the process of developing and implementing interventions to reduce readmission rates and improve hospital quality of care. Nevertheless, despite the intense interest in readmissions, there is limited understanding of how to prognosticate and mitigate risk for readmissions based on predischarge factors. In that context, we sought to develop the prediction model for 5-years readmission of patients diagnosed with schizophrenia and schizoaffective (SZ/SA) disorders.

The readmission rates have ranged from $14 \%$ to $80 \%$ in adults with SZ/SA disorders that may be due to differences in the study period: from 3 - 6 months up to 3 years [1,2]. There is a group with high-service-utilizing patients [3]. For example, $12 \%$ of 307 schizophrenia patients were frequent users of hospitalization - three or more admissions within a 30-month period [4].

Previous studies have identified risk factors associated with hospital readmission among adults with severe mental disorders $[1,2,5]$. However, these studies have been limited by a number of factors, including unmarried status, clinical variables, previous psychiatric hospitalizations, a duration of index hospitalization, alcohol abuse, a diagnosis of severe mental disorder [6], suicidal ideation or plans [7], agitation of patients [8], and involuntary admission [9]. However, studies using patient-reported outcome measures (PROMs) [10] are scanty. Indeed, predictive role of predischarge measured psychological distress, perceived social support, health-related quality of life (QOL), and functional status has not been specifically investigated. Further, no studies have examined the concurrent attribution of demographic, clinical, and psychosocial variables to the readmission rates over five years after index-discharge. In addition, none has developed a prediction model to identify high readmission risk patients before discharge.

The present study was undertaken to identify predischarge predictors of hospital readmissions of persons with SZ/SA disorders using PROMs. Specifically, based on the Distress/Protection Vulnerability model [11], our analysis included demographic (age and gender), background, and clinical characteristics together with three PROMs: psychological distress (with harmful or distressing effect), perceived social support (with putative protective or enhancing effect), and perceived QOL. The following questions were addressed.
- What is the frequency of readmissions in patients with SZ/SA disorders over 5 years after discharge from index hospitalization?

- Do readmit and non-readmitted patient groups differ in terms of demographics and background features, clinical and PROMs?

- What are the predischarge predictors for post-discharge readmission?

We hypothesized that hospital readmission would be associated with PROMs rather than demographic and illness-related variables among persons with SZ/SA disorders.

\section{Materials and Methods}

\section{Study design and population}

This study is a part of the prospective project titled: "Risk factors for hospital readmission in schizophrenia and schizoaffective patients". This study was carried out at the Shaar Menashe Mental Health Center, a public university teaching hospital in Israel. A detailed description of the project design, data collection, measures, and baseline findings were reported previously [12,13]. Briefly, inpatients were identified at five participating wards in the predischarge period (2-3 weeks before discharge). Each patient was introduced to a researcher, who provided a full explanation of the study and asked for a written study consent. Eligible patients included adult men or women (aged 18-65 years) having DSM-5 SZ/ SA disorders [14], and receiving inpatient psychiatric care. The exclusion criteria for participants were: different diagnosis than SZ/ SA disorders, serious neurologic disorders, dementia, mental retardation, and pregnancy. The readmissions were tracked via computerized medical records over 5 years after an index discharge - the primary outcome variable. The study was approved by the Shaar Menashe Internal Review Board. Written informed consent was obtained from all eligible patients.

\section{Participants}

A total of 174 inpatients were recruited over an 18-month period (during 2014-2015 years), with a response rate of $71.8 \%$. Among the 125 participants, 96 (76.8\%) were male. The mean age was $39.6 \pm 11.4$ years (range: $20-62$ ). Most of the sample (77 of 125 , or $61.6 \%$ ) were single; a few (11 or $8.8 \%$ ) were married, and the rest (37 or $29.6 \%$ ) were divorced, separated, or widowed. 
The mean level of educational attainment was $10.9 \pm 2.2$ years. The mean age of patients applying for psychiatric care was $24.7 \pm 8.7$ years, and the mean duration of the disorder was $14.6 \pm 9.7$ years (range: 2-40).

\section{Measures}

All participants were assessed in a stable state before discharge from the hospital. Assessments were administered by trained clinical raters. The diagnosis was based on a face-to-face interview, medical records, and a consensus between two senior psychiatrists. Sociodemographic and background variables, such as age at examination, gender, marital status, educational level, age when patients first applied for psychiatric care, and illness duration, were collected.

Illness severity at the time of discharge was evaluated using the Clinical Global Impression-Severity (CGI-S) scale, which ranges from 1 (normal) through to 7 (among the most severely ill) [15].

The Mini Positive and Negative SyndromeScale (Mini-PANSS) was used to assess the severity of psychopathology with high scores indicating more severe symptoms [16]. The subscales of the MiniPANSS can be linked to scores on the original PANSS subscales with very little bias. Inter-rater reliability scores for the mini-PANSS ratings in our sample were 0.85-0.90.

The Global Assessment of Functioning (GAF) scale was used for measures of impairment and functioning [17].

Suicide risk was assessed using the Plutchik Suicide Risk Scale (PSRS) [18]. The PSRS is a 24-item self-report measure with "yes" or "no" responses. Scores range from 0 to 24 with high scores indicating increased suicidal risk. The measure has good reliability and validity, and it can be used in the psychiatric and non-psychiatric groups [18]. Cronbach's $\alpha$ in our samples was 0.82 .

Aggressiveness was assessed by The Overt Aggression Scale (OAS) [19]. This is a four-part behavior rating scale used to evaluate and document the frequency and severity of aggressive episodes: verbal aggression, physical aggression against objects, physical aggression against self, and physical aggression against others. A total mean score at the last month was used in this study.

Compliance with treatment was examined by the Brief Adherence Rating Scale (BARS) [20]. It is a pencil-paper, clinician- administered adherence assessment which consists of 4items: 3 questions and an overall visual-analog rating scale to assess the proportion of doses taken by the patient in the past month $(0 \%$ $-100 \%)$. Compliance was defined as none, partial or full.

Emotional distress was assessed using the Talbieh Brief Distress Inventory (TBDI) [21,22]. The TBDI is a 24-item self-report instrument that measures subjective discomfort from psychiatric symptoms. Responses range from 0 to 4 , with high scores indicating the great intensity of six distress dimensions - symptoms (obsessiveness, hostility, sensitivity, depression, anxiety, and paranoid ideation). Cronbach's $\alpha$ for TBDI symptoms ranged from 0.76 to 0.91 in this study.

The Multidimensional Scale of Perceived Social Support (MSPSS) was used to assess emotional support and the degree of satisfaction with perceived social support from family, friends, and significant others [23]. The higher the score, the higher the perceived social support. Cronbach's $\alpha$ for the total score and tree dimensions ranged from 0.84 to 0.91 .

The Quality of Life Enjoyment and Satisfaction Questionnaire -Abbreviated version (Q-LES-Q-18) was used to assess the healthrelated QOL indexes and domains: physical health, subjective feeling, leisure time activity, social relationship, and satisfaction with medication [24]. Responses were scored on a 5-point scale, and higher scores indicated better QOL with specific life domains. Internal consistency measured by Cronbach's $\alpha$ coefficient ranged from 0.82 to 0.92 .

All questionnaires were translated into Hebrew and back-translated into English by an independent translator, and these questionnaires were also used in previous studies [11,13].

\section{Statistical analysis}

Three domains of predischarge variables were used for analyses:

- Demographic and background variables: age, gender, marital status, educational level (years), age of onset (years), illness duration (years), DSM-5 diagnosis, lifetime previous hospitalizations, the status of index admission, antipsychotic prescription at discharge, compliance with treatment, and reasons for discharge; 
- Clinical characteristics measured with CGI-S, MiniPANSS, PSRS, GAF, and OAS;

- Patient-reported outcome variables measured with TBDI, MSPSS, and Q-LES-Q.

A single individual could have multiple readmissions over the study period. Two admissions with less than a 14-day interval were treated as a single admission.

Frequent users were defined as having a number of readmissions more than one standard deviation above the average for the whole sample.

According to readmittance status, the participants were divided into two groups: 'non readmitted' versus 'readmitted', and into three groups: 'non readmitted', 'readmitted', and 'readmitted with long-term hospitalization' (the subjects who were hospitalized for $>1$ year).

The statistical analysis was performed using the Number Cruncher Statistical Systems [25] in four steps as follows.

- Descriptive statistics were used to describe all sample data. Continuous variables were presented by mean and standard deviation (SD). Internal consistency was evaluated by means of Cronbach's $\alpha$. Differences in variables were examined with the $\chi^{2}$-test for equality of proportions for categorical variables, and t-test, or the Wilcoxon signed-rank test(z) - for assessing continuous variables. All tests were two-sided, and $\alpha$ was set at $\mathrm{p}<$ 0.05 for all hypotheses.

- The three patient subgroups ('readmitted', 'non readmitted', and 'readmitted with long-term hospitalization') were compared by each independent variable using one-way analysis of variance (ANOVA).

The significant predictors for readmission were explored with multivariate multiple regression analysis with stepwise backward selection. In constructing this model, the number of admissions of each patient during the study period was used as a dependent variable. A set of independent variables was used for the variable selection procedure: age, education, age of onset, illness duration, lifetime admissions, CGI-S, Mini-PANSS, PSRS, GAF, OAS, TBDI, MSPSS, and Q-LES-Q-18 domains. An adjusted $\mathrm{R}^{2}$ was computed to evalu- ate the ratio of the sum of squares explained by a regression model. The Wald statistic and confidence interval were used to assess the significance of individual logistic regression coefficients. The effect size (Cohen's $\mathrm{f}^{2}$ ) was also calculated (https://www.analyticscalculators.com/calculator.aspx?id=5).

To determine predictors, we also performed backward stepwise multiple logistic regression using the dichotomous variable ('nonreadmitted' versus 'readmitted' persons). For the step-wise selection procedure, we used both continuous and categorical variables (see Tables 1 and 2). By logistic regression analyses, we estimated odds ratios (OR) for particular variables, controlling for the other variables. Diagnostic test evaluation was performed based on findings obtained from the logistic regression model (http://www.vassarstats.net/clin1.html). Quantities typically used to evaluate the prognostic accuracy of binary variables are sensitivity, specificity, positive predictive values (PPV), and negative predictive values (NPV), positive likelihood ratio (+LR), and negative likelihood ratio (-LR) (see Table 5 for definitions). An assessment of the overall prognostic accuracy is typically assessed by the area under the corresponding receiver operating characteristic (ROC) curve [26]. A slightly lower than required value of sensitivity for symptoms $(\geq 0.25)$ and a value of PPV $(\geq 0.70)$ were chosen as selection criteria.

\section{Results}

\section{Sample characteristics}

One hundred twenty-five clinically stable inpatients with either schizophrenia $(n=81)$ or schizoaffective disorders $(n=44)$ participated in this study. All patients were medicated and treated with first- and second-generation antipsychotic agents. None of the participants had to exacerbate physical disorders during the assessment.

\section{Patterns of readmissions}

Readmission rates in the study period were evaluated in the following fashion.

- In the five-year period, 125 patients accounted for 515 admissions. The mean number of admissions per patient was $4.1 \pm 2.2$ (ranged from 0 to 25 ).

- During the first year, 79 of 125 (63.2\%) patients were readmitted to the hospital, at the second year of study - additional 14 (11.2\%) people, at the third year - 4 
(3.2\%), and at the fourth year - 4 (3.2\%). Thus, two-year readmission rate was $74.4 \%$ (93 patients), three-year rate $-77.6 \%$ (97patients).

- Overall, for the 5 -year period $80.8 \%$ had at least one readmission ('readmitted group' = 101 patients). Consequently, 24 patients (19.2\%) were not rehospitalized for the 5-year period ('non-readmitted group').

- Since a single individual could have multiple readmissions over the course of the study, every-year readmission rate ranged from 43.2 to $63.2 \%$ in 125 subjects, particularly, at the 1-st year $79(63.2 \%)$ patients were readmitted; at the 2-nd year - 74 (59.2\%), at the 3-rd year - $60(48.0 \%)$, at the 4-th year - $59(47.2 \%)$ and at the 5 -th year - 54 (43.2\%).

- Frequent users were defined as having admissions more than one standard deviation (SD) above the average $(4.1 \pm 2.2=6.3)$, meaning at least 6 readmissions in the 5-year period. The frequent users accounted for $26(20.8 \%)$ of 125 patients who were readmitted $8.2 \pm$ 5.9 times for the study period (compared with $3.0 \pm 2.7$ times for the remaining 99 patients, $\mathrm{p}<0.001$ ). These 26 frequent users were responsible for $41.6 \%$ (214 of 515 ) of admissions during the study period. Most of these patients, 20 of 26 patients (76.9\%), were admitted voluntarily to the index hospitalization.

\begin{tabular}{|c|c|c|c|c|c|c|c|c|}
\hline \multirow[t]{2}{*}{ Variables } & \multicolumn{2}{|c|}{$\begin{array}{c}\text { Non-readmitted } \\
\qquad \mathrm{N}=24\end{array}$} & \multicolumn{2}{|c|}{$\begin{array}{l}\text { Readmitted to hospital } \\
\qquad N=74\end{array}$} & \multicolumn{2}{|c|}{$\begin{array}{l}\text { Long-term hospitalized } \\
\qquad N=27\end{array}$} & \multicolumn{2}{|c|}{ Significance } \\
\hline & $\mathrm{N}$ & $\%$ & $\mathrm{~N}$ & $\%$ & $\mathrm{~N}$ & $\%$ & $x^{2}$ & $\mathrm{p}$ \\
\hline Gender male & 17 & 70.8 & 53 & 71.6 & 26 & 96.3 & 7.3 & 0.025 \\
\hline Females & 7 & 29.2 & 21 & 28.4 & 1 & 3.7 & $\mathrm{df}=2$ & \\
\hline \multicolumn{9}{|l|}{ Marital status } \\
\hline Never married & 16 & 66.7 & 46 & 62.2 & 15 & 55.6 & 1.1 & 0.89 \\
\hline Married & 2 & 8.3 & 7 & 9.5 & 2 & 7.4 & $\mathrm{df}=4$ & \\
\hline Unmarried $^{1}$ & 6 & 25.0 & 21 & 28.4 & 10 & 37.0 & & \\
\hline \multicolumn{9}{|l|}{ Diagnosis (DSM-5) } \\
\hline Schizophrenia & 12 & 50.0 & 48 & 64.9 & 21 & 77.8 & 4.31 & 0.11 \\
\hline Schizoaffective disorder & 12 & 50.0 & 26 & 35.1 & 6 & 22.2 & $\mathrm{df}=2$ & \\
\hline \multicolumn{9}{|l|}{ Status of index admission: } \\
\hline Voluntary & 7 & 29.2 & 26 & 35.1 & 5 & 18.5 & 11.0 & 0.026 \\
\hline Involuntary & 12 & 50.0 & 30 & 40.5 & 7 & 25.9 & $\mathrm{df}=4$ & \\
\hline Court-ordered & 5 & 20.8 & 18 & 24.3 & 15 & 55.6 & & \\
\hline \multicolumn{9}{|l|}{ Antipsychotic therapy } \\
\hline Monotherapy & 10 & 41.7 & 29 & 39.2 & 11 & 40.7 & 0.05 & 0.97 \\
\hline Polypharmacy² & 14 & 58.3 & 45 & 60.8 & 16 & 59.3 & $\mathrm{df}=2$ & \\
\hline \multicolumn{9}{|c|}{ Compliance with treatment, } \\
\hline BARS None & 1 & 4.2 & 10 & 13.5 & 1 & 3.7 & 7.7 & 0.10 \\
\hline Partial & 11 & 45.8 & 46 & 62.2 & 17 & 63.0 & $\mathrm{df}=4$ & \\
\hline Full & 12 & 50.0 & 18 & 24.3 & 9 & 33.3 & & \\
\hline \multicolumn{9}{|l|}{ Reasons for discharge } \\
\hline No improvement & 6 & 25.0 & 25 & 33.8 & 18 & 66.7 & 11.5 & 0.003 \\
\hline Clinical improvement & 18 & 75.0 & 49 & 66.2 & 9 & 33.3 & $\mathrm{df}=2$ & \\
\hline
\end{tabular}

Table 1: Comparisons between patients with different readmittance statuses over 5 years after index-discharge.

1) Divorced, separated, widowed. 2) The use of two or more antipsychotic medications. 


\section{Univariate analysis}

Tables 1 and 2 compare the non-readmitted $(\mathrm{N}=24)$, readmitted $(\mathrm{N}=74)$, and readmitted with long-term hospitalization $(\mathrm{N}=$ 27) patients by key variables. At a univariate level, individuals who were readmitted with long-term hospitalization had a higher pro- portion of males $(96.3 \%, \mathrm{p}<0.05)$, the court-ordered legal status of index admission (55.6\%, $\mathrm{p}<0.05)$, no improvement at discharge $(66.7 \%, \mathrm{p}<0.005)$. The patients - frequent users reported higher paranoid ideations scores: $1.6 \pm 1.0$ versus non-readmitted and versus long-term hospitalized patients $(\mathrm{p}<0.05)$.

\begin{tabular}{|c|c|c|c|c|c|c|c|c|c|c|}
\hline \multirow[t]{2}{*}{ Variables } & \multicolumn{2}{|c|}{$\begin{array}{l}\text { Not readmitted } \\
\qquad \mathrm{N}=\mathbf{2 4}\end{array}$} & \multicolumn{2}{|c|}{$\begin{array}{l}\text { Readmitted } \\
\text { to hospital } \\
\mathrm{N}=74\end{array}$} & \multicolumn{2}{|c|}{$\begin{array}{l}\text { Significance } \\
\text { t (z) - test }\end{array}$} & \multicolumn{2}{|c|}{$\begin{array}{c}\text { Long-term } \\
\text { hospitalized } \\
\text { N }=27\end{array}$} & \multicolumn{2}{|c|}{$\begin{array}{c}\text { Significance } \\
\text { ANOVA } \\
\text { df }=2,125\end{array}$} \\
\hline & Mean & SD & Mean & SD & $t / z$ & p & Mean & SD & $\mathbf{F}$ & p \\
\hline Age, years & 42.6 & 13.0 & 38.9 & 11.4 & 1.3 & 0.20 & 38.8 & 9.6 & 1.0 & 0.35 \\
\hline Education, years & 11.3 & 1.3 & 10.8 & 2.4 & 1.3 & 0.18 & 10.7 & 2.5 & 0.6 & 0.56 \\
\hline Age of onset, years & 26.3 & 10.8 & 24.1 & 7.9 & 0.8 & 0.42 & 25.0 & 9.2 & 0.6 & 0.56 \\
\hline Illness duration, years & 14.9 & 11.5 & 14.1 & 9.3 & 0.3 & 0.73 & 15.5 & 9.3 & 0.2 & 0.81 \\
\hline Illness severity, CGI-S & 4.5 & 0.9 & 4.5 & 0.9 & 0.03 & 0.97 & 4.7 & 1.1 & 0.2 & 0.84 \\
\hline \multicolumn{11}{|l|}{ Symptom severity, } \\
\hline Mini-PANSS & 45.3 & 10.2 & 46.1 & 11.8 & 1.5 & 0.62 & 44.6 & 12.2 & 0.2 & 0.85 \\
\hline Positive & 12.5 & 3.8 & 12.8 & 5.8 & 0.2 & 0.83 & 11.7 & 4.8 & 0.4 & 0.12 \\
\hline Negative & 16.3 & 5.2 & 16.9 & 5.4 & 0.5 & 0.62 & 16.8 & 5.1 & 0.1 & 0.88 \\
\hline General & 14.8 & 3.7 & 14.7 & 4.1 & 0.1 & 0.93 & 14.6 & 5.1 & 0.02 & 0.98 \\
\hline Functioning, GAF & 49.0 & 13.9 & 48.1 & 12.3 & 0.3 & 0.76 & 47.6 & 13.3 & 0.1 & 0.92 \\
\hline Aggressiveness, OAS & 0.9 & 1.1 & 0.9 & 1.1 & 0.3 & 0.78 & 0.8 & 1.2 & 0.2 & 0.81 \\
\hline Suicide risk, PSRS & 6.8 & 4.2 & 8.8. & 4.4 & 2.0 & 0.050 & 7.2 & 3.5 & 2.8 & 0.61 \\
\hline Emotional distress, TBDI & 1.0 & 0.6 & 1.2 & 0.8 & 1.2 & 0.21 & 0.9 & 0.7 & 1.9 & 0.15 \\
\hline Obsessiveness & 1.2 & 1.2 & 1.2 & 1.2 & 1.1 & 0.80 & 1.1 & 1.0 & 0.04 & 0.96 \\
\hline Sensitivity & 1.1 & 0.9 & 1.4 & 1.0 & 1.6 & 0.13 & 1.0 & 0.9 & 2.5 & 0.089 \\
\hline Depression & 1.1 & 0.8 & 1.2 & 1.0 & 0.4 & 0.66 & 0.9 & 1.0 & 0.7 & 0.16 \\
\hline Anxiety & 0.7 & 0.8 & 1.0 & 1.0 & 1.3 & 0.19 & 0.8 & 0.9 & 0.9 & 0.42 \\
\hline Hostility & 0.4 & 0.5 & 0.9 & 1.1 & 3.2 & 0.002 & 0.7 & 0.9 & 2.7 & 0.073 \\
\hline Paranoid ideations & 1.3 & 1.1 & 1.6 & 1.1 & 1.2 & 0.21 & 0.9 & 0.9 & 4.9 & 0.009 \\
\hline Social support, MSPSS & 60 & 17.8 & 54.3 & 18.6 & 1.2 & 0.22 & 61.5 & 17.2 & 1.9 & 0.15 \\
\hline Family support & 20.7 & 8.4 & 18.7 & 7.9 & 1.0 & 0.29 & 20.6 & 7.4 & 0.9 & 0.40 \\
\hline Friend support & 17.0 & 8.1 & 15.4 & 8.9 & 0.8 & 0.43 & 19.2 & 7.3 & 2.0 & 0.13 \\
\hline Other significant support & 22.4 & 5.9 & 20.3 & 6.6 & 1.4 & 0.18 & 21.7 & 5.8 & 1.2 & 0.32 \\
\hline $\begin{array}{l}\text { Quality of life, Q-LES- } \\
\text { Q-18 }\end{array}$ & 3.8 & 0.9 & 3.7 & 0.9 & 0.6 & 0.52 & 3.9 & 0.7 & 0.9 & 0.40 \\
\hline Subjective feeling & 3.9 & 1.0 & 3.9 & 0.9 & 0.2 & 0.84 & 4.1 & 0.9 & 0.8 & 0.47 \\
\hline Leisure time activities & 4.2 & 1.0 & 3.8 & 1.2 & 1.5 & 0.14 & 4.0 & 0.9 & 1.4 & 0.25 \\
\hline Social relationship & 3.7 & 1.2 & 3.5 & 1.2 & 0.7 & 0.47 & 3.7 & 1.0 & 0.4 & 0.66 \\
\hline Lifetime admissions & 6.1 & 5.0 & 12.4 & 10.8 & 2.8 & 0.001 & 11.7 & 14.7 & 3.1 & 0.049 \\
\hline
\end{tabular}

Table 2: Comparison of predischarge dimensional variables between groups of patients according to readmittance status over five years after discharge.

*) z-test between 'readmitted to hospital' group and 'long-term hospitalized' patients. 
On the contrary, non-readmitted persons showed lower scores on TBDI hostility (0.4 vs.0.9, $\mathrm{p}<0.005)$ and suicide risk scale $(6.8$ vs. 8.8, $\mathrm{p}<0.05$ ), and fewer life time admissions (6.1 vs. $12.4, \mathrm{p}$ $<0.001$ ) than readmitted persons. No significant differences were found between the compared groups in terms of age, marital status, education, illness duration, diagnosis, illness and symptom severity, antipsychotic therapy, compliance with treatment, TBDI, GAF, OAS, Q-LES-Q, and MSPSS scores.

\section{Predicting the number of readmissions}

Table 3 displays a summary of the multiple regression model to predict the number of hospital readmissions in a whole sample.
This model provides the best fit to the data and it has revealed five predictors. Two predictors with a negative effect are the number of lifetime admissions ( $\mathrm{p}<0.05)$ and hostility scores ( $\mathrm{p}<0.005)$, and three predictors with a protective effect: subjective feeling $(\mathrm{p}<$ 0.001 ), social relationship, and leisure time activity (both $p<0.05$ ). By other words, the model suggests that history of previous hospitalization, elevated hostility scores, dissatisfaction with subjective feeling, social relationship, and leisure time activity scores significantly predicted recurrent readmissions after discharge. This model accounted for $23 \%$ of the variance with adjusted $R^{2}=0.19, F_{5,125}$ $=5.9$, and $\mathrm{p}<0.001$.

\begin{tabular}{|l|c|c|c|c|c|}
\hline Independent variables & $\mathbf{b}$ & $\mathbf{t}$ & $\mathbf{P}$ & Effect size (f) & $\mathbf{R}^{2} \mathbf{( \% )}$ \\
\hline $\begin{array}{l}\text { Number of lifetime } \\
\text { admissions }\end{array}$ & 0.09 & 2.1 & 0.043 & 0.11 & 9.8 \\
\hline $\begin{array}{l}\text { TBDI } \\
\text { distress - hostility }\end{array}$ & 0.58 & 2.7 & 0.005 & 0.04 & 3.1 \\
\hline $\begin{array}{l}\text { Q-LES-Q-18, } \\
\text { satisfaction with }\end{array}$ & -1.18 & 3.5 & 0.001 & 0.06 & 5.9 \\
\hline Subjective feeling & -0.72 & 2.0 & 0.049 & 0.04 & 3.9 \\
\hline Social relationship & -0.58 & 1.9 & 0.049 & 0.02 & 1.9 \\
\hline $\begin{array}{l}\text { Leisure time } \\
\text { activities }\end{array}$ & & & & \\
\hline
\end{tabular}

Table 3: Summary of multiple regressions to predict the number of hospital readmissions during the study period from predischarge variables of 125 patients with schizophrenia and schizoaffective disorder.

Only significant predictors were presented; $\beta$ - is a standardized regression coefficient. Partial $\mathrm{R}^{2}$ reflects the percentage of variation in the readmission rates explained by each independent variable adjusted to the effects of rest independent variables; $\mathrm{f}^{2}$ - effect size for multiple regression: $\mathrm{f}^{2}$ values near 0.02 are defined as small; 0.10 - 0.15 - as a medium; and above 0.35 - as large [48]. Abbreviations:

TBDI - Talbieh Brief Distress Inventory; Q-LES-Q - 18 - Quality of Life Enjoyment and Life Satisfaction Questionnaire.

\section{Predicting readmitted patients}

In general, multivariate logistic regression analysis was employed to model the outcomes of a categorical dependent variable (readmission at 0 and 1 level; ' 0 ' for non-readmitted group and ' 1 ' for readmitted ones). The use of a linear regression model for categorical variables was considered inappropriate because the response values were not measured, and the error terms were not normally distributed. The best-fitted logistic model identified ten significant variables for discrimination readmitted and non-readmitted patients, as shown in table 4.

A history of previous hospitalization, elevated suicide risk, sensitivity, and hostility TBDI scores showed the strongest association with readmitted persons (OR: 1.1, 1.3, 2.8, and 3,1, respectively). 


\begin{tabular}{|c|c|c|c|c|c|c|c|c|}
\hline \multirow[b]{2}{*}{ Measures } & \multirow{2}{*}{$\begin{array}{c}\text { Predischarge } \\
\text { variables }\end{array}$} & \multirow[b]{2}{*}{$\beta$} & \multirow[b]{2}{*}{ SE } & \multirow{2}{*}{$\begin{array}{c}\text { Wald } \\
\text { z-value } \\
(\beta=0)\end{array}$} & \multirow[b]{2}{*}{$\mathbf{p}$} & \multicolumn{3}{|c|}{ Odds Ratios (OR) ${ }^{a}$} \\
\hline & & & & & & OR & $\begin{array}{l}\text { Lower } \\
95 \% \text { CI }\end{array}$ & $\begin{array}{l}\text { Upper } \\
95 \% \text { CI }\end{array}$ \\
\hline & $\begin{array}{c}\text { Number of lifetime } \\
\text { admissions }\end{array}$ & 0.12 & 0.04 & 2.39 & 0.016 & 1.1 & 1.0 & 1.2 \\
\hline PSRS & Suicide risk & 0.24 & 0.12 & 1.96 & 0.049 & 1.3 & 1.0 & 1.6 \\
\hline \multirow[t]{4}{*}{ TBDI } & Sensitivity & 1.03 & 0.47 & 2.15 & 0.031 & 2.8 & 1.1 & 7.2 \\
\hline & Hostility & 1.14 & 0.52 & 2.18 & 0.029 & 3.1 & 1.1 & 8.8 \\
\hline & Depression & -1.01 & 0.53 & 1.89 & 0.050 & 0.4 & 0.1 & 1.0 \\
\hline & Paranoid ideations & -0.85 & 0.35 & 2.37 & 0.017 & 0.4 & 0.2 & 0.9 \\
\hline \multirow[t]{2}{*}{ Q-LES-Q-18 } & Subjective feelings & -1.08 & 0.60 & 1.96 & 0.049 & 3.0 & 0.9 & 9.7 \\
\hline & $\begin{array}{c}\text { Leisure time } \\
\text { activities }\end{array}$ & -0.82 & 0.43 & 1.99 & 0.048 & 0.4 & 0.2 & 1.0 \\
\hline MSPSS & $\begin{array}{c}\text { Other significant } \\
\text { support }\end{array}$ & -0.13 & 0.05 & 2.49 & 0.012 & 0.9 & 0.8 & 1.0 \\
\hline BARS & $\begin{array}{l}\text { Compliance with } \\
\text { treatment }\end{array}$ & -0.76 & 0.37 & 2.04 & 0.040 & 0.5 & 0.2 & 1.0 \\
\hline
\end{tabular}

Table 4: Summary of multivariate logistic regression to predict risk for 5-year readmissions from predischarge variables.

Model's properties: Dependent variable - readmission state, two groups - readmitted versus non-readmitted patients. The non-readmitted patient group was a reference group (in the analysis, the readmitted patient group was compared to this group). The odds ratio is associated with regression coefficient ( $\beta$ ). SE - the standard error; CI - confidential interval Abbreviations: PSRS - Plutchik Suicide Risk Scale, TBDI - Talbieh Brief Distress Inventory, Q-LES-Q - 18 - Quality of Life Enjoyment and Satisfaction Questionnaire - Abbreviated version; MSPSS - Multidimensional Scale of Perceived Social Support, BARS - Brief Adherence Rating Scale.

On the contrary, six protective predictors were associated with non-readmitted patients. Particularly, they felt lower depression and paranoid ideations (TBDI), were satisfied with subjective feelings, leisure time activities (Q-LES-Q-18), and other significant support (MSPSS), showed adherence to treatment (actively participated in treatment plans; BARS).

This model accounted for $31 \%$ of the variance $\left(R^{2}=0.31\right)$, df $=10$, likelihood iterations $=7$, maximum iterations $=1000$; final log-likelihood $=-53.2371$. The obtained model correctly classified $83.1 \%$ of 125 subjects.
Diagnostic test evaluation was performed, based on findings obtained from the final logistic regression model. As can be seen in Table 5, classifying the readmission state as 'readmitted' - or 'non-readmitted'- provided a sensitivity of $85.4 \%$, specificity of 46.7\%, PPV 92.2\%, NPV 30.4\%, clinically important +LR 11.75, and the small difference in -LR 2.28. The receiver operating characteristic (ROC) curves predicted readmitted state over the 5-year period, as plotted in figure 1. The ROC curves were used to find the best cut-off points for classification, which means that $83 \%$ of the classification of 125 patients was correct with this regression model. 


\begin{tabular}{|c|c|c|c|}
\hline \multirow{2}{*}{ Parameters } & \multirow{2}{*}{$\begin{array}{l}\text { Estimated } \\
\text { Value }\end{array}$} & \multicolumn{2}{|c|}{$\begin{array}{l}\text { 95\% Confidence } \\
\text { Interval }\end{array}$} \\
\hline & & $\begin{array}{l}\text { Lower } \\
\text { Limit }\end{array}$ & $\begin{array}{l}\text { Upper } \\
\text { Limit }\end{array}$ \\
\hline Prevalence (\%) & 88,0 & 81.0 & 92.9 \\
\hline Sensitivity (\%) & 85.4 & 77.2 & 91.2 \\
\hline Specificity (\%) & 46.7 & 22.2 & 72.6 \\
\hline \multicolumn{4}{|c|}{ For any particular test result, the probability that it will be: } \\
\hline Positive (\%) & 81.6 & 73.4 & 87.7 \\
\hline Negative (\%) & 18.4 & 12.2 & 26.5 \\
\hline \multicolumn{4}{|c|}{ For any particular positive test result, the probability that it is: } \\
\hline $\begin{array}{l}\text { True positive } \\
\text { (Positive Predictive Value) }\end{array}$ & 92.2 & 84.7 & 96.3 \\
\hline False positive & 7.8 & 3.7 & 15.3 \\
\hline \multicolumn{4}{|c|}{ For any particular negative test result, the probability that it is: } \\
\hline $\begin{array}{l}\text { True negative } \\
\text { (Negative Predictive Value) }\end{array}$ & 30.4 & 14.0 & 53.0 \\
\hline False negative & 69.6 & 47.0 & 85.9 \\
\hline \multicolumn{4}{|c|}{ Likelihood Ratios (weighted by prevalence) } \\
\hline $\begin{array}{l}\text { Positive Likelihood Ratio } \\
(+\mathrm{LR})\end{array}$ & 11.75 & 6.03 & 22.91 \\
\hline $\begin{array}{l}\text { Negative Likelihood Ratio } \\
\text { (-LR) }\end{array}$ & 2.28 & 1.44 & 3.64 \\
\hline
\end{tabular}

Table 5: Diagnostic test evaluation for the prediction of 5-year hospital readmissions based on a final logistic regression model. The sensitivity was a correctly identified 'readmitted state' (true positive rate), and the specificity was a correctly identified 'nonreadmitted state' (true negative rate). Positive predictive value (PPV) is probability that the 'readmitted state' is present when the test is positive. Negative predictive value (NPV) is probability that the 'non readmitted state' is not present when the test is negative. Positive likelihood ratio ( $+\mathrm{LR}$ ) is a ratio between the probability of a positive test result given the presence of the 'readmitted state' and the probability of a positive test result given the absence of the 'readmitted state', i.e. it is true positive rate/false positive rate or sensitivity/(1-specificity). Negative likelihood ratio (-LR) is ratio between the probability of a negative test result given the presence of the 'non readmitted state' and the probability of a negative test result given the absence of the 'readmitted state', i.e. equals false negative rate/true negative rate or (1-sensitivity)/ specificity.

\begin{tabular}{|l|c|c|c|}
\hline \multirow{2}{*}{} & \multicolumn{2}{|c|}{ Condition } & \multirow{2}{*}{ Totals } \\
\cline { 2 - 3 } & Absent & Present & \\
\hline Test positive & 8 & 94 & 102 \\
\hline Test negative & 7 & 16 & 23 \\
\hline Totals & 15 & 110 & 125 \\
\hline
\end{tabular}

Table a: Using these entered data, midpoints and 95\% confidence intervals (CI) were calculated. Entered data from regression model.

\section{Discussion}

Readmission rates are considered a proxy indicator of the adequacy of mental health care [27]. In this study, we assume that a set of PROMs would be useful for predicting readmissions of patients with SZ/SA disorders. As expected, post-discharge readmission was significantly associated with TBDI, MSPSS, and Q-LES-Q-18 measures rather than with demographic and illness-related variables among persons with SZ/SA disorders. This study addressed three specific questions.

The first research question addressed the frequency of readmissions of patients with SZ/SA disorders over 5 years after a dis- 
charge from an index hospitalization. The present study showed that about $63.2 \%$ of the readmissions occur within the first year after discharge, and $80.8 \%$ - within the study period of 5 years. Rather, since a single individual could have multiple readmissions over the course of the study, the annual readmission rate escalated from 43.2 to $63.2 \%$. These findings are in line with previously published results (from $50 \%$ to $84 \%$ ) [28,29]. Furthermore, frequent users accounted for $20.8 \%$ of patients that also confirm published rates (20 - 29\%) [30,31]. Thus, the descriptive findings indicated that our sample included individuals with reasonable patterns of readmissions and may be suitable for testing predictors.

The second research question addressed the differences between 'readmitted' and 'non- readmitted' patient groups in terms of demographics and background features, clinical and psychosocial variables. In order to answer this question, $19.2 \%$ of patients were defined as 'non-readmitted group', 59.2\% -as 'readmitted group', and $21.6 \%$ - as 'readmitted patients with long-term hospitalization'. Non-readmitted patients showed lower scores on hostility and suicide risk, fewer lifetime admissions, than those with readmittance status. Readmitted patients with long-term hospitalization had a higher proportion of males (96.3\%, p < 0.05), courtordered legal status of an index admission (55.6\%, p < 0.05), lacking improvement at discharge (66.7\%, p < 0.005; Table 1).

The third research question addressed the predischarge predictors - reasons for post discharge admission. In order to answer this question, multiple regression and multivariate logistic regression analyses were carried out with the number of variables (Tables 1 and 2). The multiple regression model for prediction of 5-year readmissions revealed two predischarge predictors with negative effects (number of lifetime admissions, and hostility), and three with protective effects (satisfaction with subjective feeling, social relationship, and leisure time activity scores). The multivariate logistic regression model correctly classified $83.1 \%$ of 125 subjects from two groups: readmitted versus non-readmitted patients. The model suggests the strongest association of a history of previous hospitalization, elevated suicide risk, sensitivity, and hostility scores with readmittance status. On the opposite, six protective predictors were significantly associated with the 'non-readmission group' of patients. They felt lower depression and paranoid ideations, reported satisfaction with subjective feelings and leisure time activities, had other significant support, and actively participated in treatment plans. It should be taken into account that the logistic regression model achieves $\mathrm{R}^{2}=0.31$. In standard linear regression, $\mathrm{R}^{2}$ gives a parameter of the power of the regressive equation: an $\mathrm{R}^{2}$ close to 1 is a very strong prediction. There is no direct equivalent of $\mathrm{R}^{2}$ for logistic regression. For the logistic model, accounts in the range of $\mathrm{R}^{2}=0.10-0.35$ are quite reasonable [32].

Next, sociodemographic, background, and clinical variables did not reach a significant level as at predischarge predictors. Published studies reported contradictory results regarding the association of sociodemographic, background, and clinical variables with hospital readmissions $[1,2]$. Unexpectedly, our study did not reveal any influence of involuntary admission or the type of discharge on the risk of readmission. Clinical factors that describe illness severity at the time of admissions, such as CGI-S, Mini-PANSS, and GAF, have not been shown to provide substantial additional value in predicting rates of hospital readmission compared to PROMs. Thus, regression analysis indicated that PROMs such as TBDI, MSPSS, Q-LES-Q-18 were important contributors to the predicting post-discharge readmission of SZ/SA disordered patients. This suggestion seems to be in line with the findings that showed consistent relationships of distress and social support with QOL of life in individuals with schizophrenia [24,33]. Heightened stress sensitivity is a common characteristic of persons with severe mental disorders which have more negative experiences in their lives, and it may be predictive of clinical and functional outcomes [34].

The findings from the present study showed that higher scores of predischarge psychological distress dimensions (sensitivity, hostility, depression and paranoid ideations) predicted more readmissions after discharge. It should be noted that elevated psychological distress in schizophrenia was found to be related to lower QOL and more suicide attempts [11,34]. The present study has found that elevated suicide risk is a significant predischarge predictor of hospital readmission. This finding aligns with the previous works $[7,35]$.

A recent meta-analysis of 100 studies has found that the suicide rate has been 4 times higher for patients who were admitted with suicidal ideation or suicidal behavior than for patients admitted for other psychiatric reasons, and 2 times higher during the first 3 months after a discharge from a psychiatric hospital [36].

It is widely recognized that social support serves as a buffer between psychological distress and QOL $[37,38]$ by attenuating or 
preventing a stressful appraisal of the situation [39]. Therefore, the role of the perceived social support in predicting hospital readmission may be very important. This finding has been consistent with the results of several other studies suggesting that lower readmission rates are associated with the protective effect of social support [40], family support [41], with living with someone (relative or caregivers) or living in an institution [42]. Thus, the use of peer mentors is a promising intervention for reducing psychiatric rehospitalizations for patients with major mental illnesses [43].

Previous studies did not deal with QOL domains for predicting hospital readmission. We found that Postrado and Lehman [44] had been mentioned dissatisfaction with family relations as a predictor of 1-year rehospitalization of patients with severe and persistent mental illness. Thus, for the first time, we have indicated the role of satisfaction with leisure time activities and subjective feelings in predicting hospital readmission.

As expected, our study has identified compliance with treatment as a significant predictor of post-discharge readmission. The finding was consistent with other studies, which demonstrated that a positive attitude toward medication decreased the risk of readmission [45-47].

Having an admission in history turned out to be significantly associated with a risk for readmission in 32 out of 37 studies, showing it as a risk factor in 31 cases [1]. As with previous studies, we found that both regression models suggest the strongest association of a history of the previous hospitalization with post-discharge readmission. Readmission risk increased with a number of previous admissions.

\section{Study limitations}

The present study has a number of limitations that need to be recognized and addressed in future studies.

First, it should be recognized that the results of the present study might apply only to individuals with chronic SZ/SA disorders (illness duration: 11-49 years) who tend to be more treatment compliant and more cooperative patients.

The second limitation is common for most studies using the self-report methodology for investigating severely ill psychiatric patients and consists of one's degree of reliability on patients' selfreports.
The third one, due to the study design, we could not assess postdischarge factors data that may have had an impact on the likelihood of rehospitalization.

However, to the best of our knowledge, this is the first study examining the predischarge patient-reported predictors standing for the readmission of patients with SZ/SA disorders. Our findings regarding the association between PROMs and readmission risk are important and intriguing. It is important because we found that distress, social support, and QOL contribute to predicting readmission in the whole sample with SZ/SA disorders. It is intriguing because the sociodemographic, background, and clinical variables did not reach a significant level as predictors of readmission risk.

\section{Conclusion}

The study is the first of its kind to investigate the role of PROMs in predicting readmissions five years after discharge. The five-year psychiatric readmission rate was $80.8 \%$ (63.2\% within the first year). The frequent users accounted for $20.8 \%$ of patients who were readmitted $8.2 \pm 5.9$ times. Patients with SZ/SA disorders who reported elevated distress sensitivity and hostility, suicide risk, and a history of the previous hospitalization were more likely to have psychiatric readmissions. The evidence demonstrates that lower depression and paranoid ideations, better satisfaction with the quality of life (subjective feelings, social relationship, and leisure time activities), social support, and adherence to treatment can reduce the risk of hospital readmissions among patients discharged to home. Our findings emphasize the importance of patient-reported outcome measures to identify patients at risk of readmission to the hospital. Since the prediction of readmission remains challenging, searching for strong predictors merits further investigations.

\section{Funding Support}

The authors have not declared a specific grant for this research from any funding agency in the public, commercial or not-for-profit sectors.

\section{Disclosure}

The authors declare that they have no conflicts of interests.

\section{Acknowledgements}

We are indebted to all of the patients who participated in this study and to the clinical and research teams of the Shaar Menashe 
Mental Health Center who helped us approach them in the different hospital wards. We are grateful to Dana Shteinberg the volunteer that helped to collect the follow-up data.

\section{Author Contributions}

Michael Ritsner: methodology, coordinated the project, formal analysis, writing- original draft preparation. Yael Ratner: acquired the data, analyzed and interpreted the data, writing- reviewing and editing of the manuscript, and approved the final version for publication.

\section{Bibliography}

1. Donisi V., et al. "Pre-discharge factors predicting readmissions of psychiatric patients: a systematic review of the literature". BMC Psychiatry 16 (2016): 449.

2. Zanardo G Lde P., et al. "Factors Associated with Psychiatric Readmissions: A Systematic Review”. Paidéia (Ribeirão Preto) 28 (2018): e2814.

3. Havassy BE and Hopkin JT. "Factors predicting utilization of acute psychiatric inpatient services by frequently hospitalized patients". Hospital and Community Psychiatry 40 (1989): 820823.

4. Roick C., et al. "Factors contributing to frequent use of psychiatric inpatient services by schizophrenia patients". Social Psychiatry and Psychiatric Epidemiology 39 (2004): 744-751.

5. Jaramillo-Gonzalez LE., et al. "The frequency of rehospitalization and associated factors in Colombian psychiatric patients: a cohort study". BMC Psychiatry 14 (2014): 161.

6. Lorine K., et al. "Risk factors associated with psychiatric readmission". The Journal of Nervous and Mental Disease 203 (2015): 425-430.

7. Bartoli F., et al. "Pre-Discharge Predictors of 1-Year Rehospitalization in Adolescents and Young Adults with Severe Mental Disorders: A Retrospective Cohort Study". Medicina (Kaunas) 56 (2020): 613.

8. Rubio-Valera M., et al. "Health service use and costs associated with aggressiveness or agitation and containment in adult psychiatric care: a systematic review of the evidence". BMC Psychiatry 4 (2015): 35.
9. Niehaus DJ., et al. "Crisis discharges and readmission risk in acute psychiatric male inpatients". BMC Psychiatry 17 (2008): $8: 44$.

10. Reininghaus U and Priebe S. "Measuring patient-reported outcomes in psychosis: conceptual and methodological review". The British Journal of Psychiatry 201 (2012): 262-267.

11. Ritsner M., et al. "Differences in quality of life domains and psychopathologic and psychosocial factors in psychiatric patients". The Journal of Clinical Psychiatry 61 (2000): 880-889.

12. Ratner Y., et al. 'Patients' satisfaction with hospital health care: Identifying indicators for people with severe mental disorder". Psychiatry Research 270 (2018): 503-509.

13. Ritsner MS and Ratner Y. "Predicting predischarge anhedonia among inpatients with schizophrenia and schizoaffective disorders: A large-scale analysis". The Journal of Nervous and Mental Disease 207 (2019): 12-21.

14. APA. "Diagnostic and statistical manual of mental disorders". 5th ed, Washington, DC: American Psychiatric Association (2013).

15. Guy W. "ECDU assessment manual for psychopharmacology". Rockville, MD: US Department of Health and Human Services (1976).

16. Khan A., et al. "Use of non-parametric item response theory to develop a shortened version of the Positive and Negative Syndrome Scale (PANSS)". BMC Psychiatry 16 (2011): 11-178.

17. GAF. "Diagnostic and statistical manual of mental disorders". American Psychiatric Association. Washington, DC. (1994).

18. Plutchik R., et al. "Correlates of suicide and violence risk: The Suicide Risk Measure". Comprehensive Psychiatry 30 (1989): 296-302.

19. Yudofsky SC., et al. "The Overt Aggression Scale for the objective rating of verbal and physical aggression". The American Journal of Psychiatry 143 (1986): 35-39.

20. Byerly MJ., et al. "The Brief Adherence Rating Scale (BARS) validated against electronic monitoring in assessing the antipsychotic medication adherence of outpatients with schizo- 
phrenia and schizoaffective disorder". Schizophrenia Research 100 (2008): 60-69.

21. Ritsner M., et al. “The Talbieh Brief Distress Inventory: A brief instrument to measure psychological distress among immigrants". Comprehensive Psychiatry 36 (1995): 448-453.

22. Ritsner M., et al. "Assessing psychological distress in psychiatric patients: Validation of the Talbieh Brief Distress Inventory". Comprehensive Psychiatry 43 (2002) :229-234.

23. Zimet GD., et al. "The Multidimensional Scale of Perceived Social Support". The Journal of Personality Assessment 52 (1988): 30-41.

24. Ritsner M., et al. "Validity of an abbreviated Quality of Life Enjoyment and Satisfaction Questionnaire (Q-LES-Q-18) for schizophrenia, schizoaffective, and mood disorder patients". Quality of Life Research 14 (2005): 1693-1703.

25. Hintze JL. NCSS 6.0. Statistical System for Windows. User's Guide. Number Cruncher Statistical Systems. Kaysville, Utah (2007).

26. Zhou X-H., et al. "Statistical methods in diagnostic medicine". New York: Wiley and Sons Interscience. (2002) 437.

27. Rumball-Smith J and Hider P. "The validity of readmission rate as a marker of the quality of hospital care, and a recommendation for its definition". The New Zealand Medical Journal 122 (2009): 63-70.

28. Bowersox NW., et al. "Predictors of Rehospitalization in HighUtilizing Patients in the VA Psychiatric Medical System". Psychiatric Quarterly 83 (2012): 53-64.

29. Boaz TL., et al. "Risk factors for early readmission to acute care for persons with schizophrenia taking antipsychotic medications". Psychiatric Services 64 (2013): 1225-1229.

30. Vogel S and Hugelet P. "Factors associated with multiple admissions to a public psychiatric hospital". Acta Psychiatrica Scandinavica 95 (1997): 244-253.

31. Graca J., et al. "Characteristics of frequent users of an acute psychiatric inpatient unit: a five-year study in Portugal". Psychiatric Services 64 (2013): 192-195.
32. Hosmer D and Lemeshow S. Applied Logistic Regression. New York: John Wiley and Sons, Inc. Mathematics, (2004). 392 pages.

33. Ho WW., et al. "Recovery components as determinants of the health-related quality of life among patients with schizophrenia: structural equation modelling analysis". Australian and New Zealand Journal of Psychiatry 44 (2010): 71-84.

34. Pallanti S., et al. "Social anxiety in outpatients with schizophrenia: a relevant cause of disability". The American Journal of Psychiatry 161 (2004): 53-58.

35. Kim HM., et al. "Intensity of outpatient monitoring after discharge and psychiatric rehospitalization of veterans with depression". Psychiatric Services 62 (2011): 1346-1352.

36. Chung DT., et al. "Suicide rates after discharge from psychiatric facilities: a systematic review and meta-analysis". JAMA Psychiatry 74 (2017): 694-702.

37. Cohen S and Wills TA. "Stress, social support and the buffering hypothesis". Psychological Bulletin 98 (1985): 310-357.

38. Ritsner M., et al. "Determinants of changes in perceived quality of life in the course of schizophrenia". Quality of Life Research 15 (2006): 515-526.

39. McColl MA., et al. "Structural relationships between social support and coping". Social Science and Medicine 41 (1995): 395407.

40. Dahlan R., et al. "Hospital-based community psychiatric service for patients with schizophrenia in Kuala Lumpur: A 1-year follow-up study of re-hospitalization". Asia-Pacific Psychiatry 5 (2013): 127-133.

41. de Castro SA., et al. "Sociodemographic and clinical characteristics of psychiatric rehospitalizations". Revista Latino-Americana de Enfermagem 18 (2010): 800-808.

42. Frick U., et al. "The revolving door phenomenon revisited: Time to readmission in 17'415 patients with 37'697 hospitalizations at a German psychiatric hospital". PLoS One 8 (2013): e75612.

43. Sledge WH., et al. "Effectiveness of peer support in reducing readmissions of persons with multiple psychiatric hospitalizations". Psychiatric Services 62 (2011): 541-544. 
44. Postrado LT and Lehman AF. "Quality of life and clinical predictors of rehospitalization of persons with severe mental illness". Psychiatric Services 46 (1995): 1161-1165.

45. Diaz E., et al. "Use of the Medication Event Monitoring System to estimate medication compliance in patients with schizophrenia". Journal of Psychiatry and Neuroscience 26 (2001): 325-329.

46. Kottsieper P. "Predicting initial aftercare appointment adherence and rehospitalization for individuals with serious mental illness discharged from an acute inpatient stay". Drexel University Publisher (Ph.D. Thesis). (2006).

47. Al-Shehhi AI., et al. "Rate and Predictors of 1-year Readmission in Tertiary Psychiatric Hospitals". Saudi Journal of Medicine and Medical Sciences 5 (2017): 224-231.

48. Cohen J. "Statistical power analysis for the behavioral sciences". 2nd ed., Lawrence Earlbaum Associates, Erlbaum, Hillsdale, NJ (1988) 567.

Volume 4 Issue 11 November 2021

(C) All rights are reserved by Yael Ratner and Michael Ritsner. 